Preprints of the

Max Planck Institute for

Research on Collective Goods

Bonn 2010/39

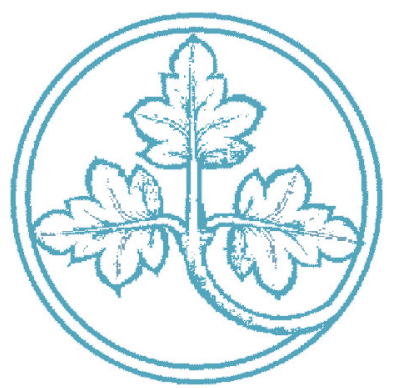

Moral Hazard and Ambiguity

Philipp Weinschenk

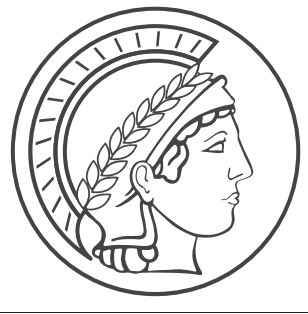




\section{Moral Hazard and Ambiguity}

Philipp Weinschenk

September 2010

Max Planck Institute for Research on Collective Goods, Kurt-Schumacher-Str. 10, D-53113 Bonn http://www.coll.mpg.de 


\title{
MORAL HAZARD AND AMBIGUITY
}

\author{
Philipp Weinschenk* \\ Bonn Graduate School of Economics \\ and Max Planck Institute for Research on Collective Goods
}

September 16, 2010

\begin{abstract}
We consider a principal-agent model with moral hazard where the agent's knowledge about the performance measure is ambiguous and he is averse towards ambiguity. We show that the principal may optimally provide no incentives or contract only on a subset of all informative performance measures. That is, the Informativeness Principle does not hold in our model. These results stand in stark contrast to the ones of the orthodox theory, but are empirically of high relevance.
\end{abstract}

JEL Classification: D82, M12, M52.

Keywords: Ambiguity Aversion, Principal-Agent Model, Moral Hazard, Informativeness Principle.

\section{INTRODUCTION}

In principal-agent models with moral hazard, the principal motivates the agent to spend effort via a performance-dependent wage scheme. Standard theory predicts (i) that the wage scheme highly depends on performance and (ii) that the principal contracts on all performance measures which are informative about the agent's effort. However, in reality, wage schemes sometimes do not depend on performance or the dependence is rather weak ${ }^{1}$ and the Informativeness Principle "seems to be violated in many occupations" (Prendergast 1999, p. 21).

* Max Planck Institute for Research on Collective Goods, Kurt-Schumacher-Str. 10, 53113 Bonn, Germany, weinschenk@coll.mpg.de. I thank Sophie Bade, Aurélien Baillon, Paul Heidhues, Martin Hellwig, Matthias Lang, Daniel Müller, and Klaas Schulze as well as seminar participants at the 4th Nordic Conference on Behavioral and Experimental Economics (2009) in Oslo and the 6th International Meeting on Experimental and Behavioral Economics (2010) in Bilbao for helpful comments and suggestions.

${ }^{1}$ See Baker, Jensen, and Murphy (1988), Jensen and Murphy (1990), Holmström and Milgrom (1991), and Prendergast (1999). 
The reason for this discrepancy between theory and empirics may be due to the following assumption: orthodox models implicitly suppose that the agent knows precisely the statistical properties of the performance measure. This is a strong assumption because "real-life choices rarely come with precise probabilities" (Post et al. 2008, p. 39). ${ }^{2}$ In this article we relax this assumption. We show that considering ambiguity and ambiguity aversion in an otherwise conventional model can bring theory in line with empirics.

The famous Ellsberg (1961) paradox predicts that individuals are averse to missing information regarding probabilities. Ellsberg suggests the following experiment. Urn A contains 50 black balls and 50 red ones. Urn B contains 100 balls, each of which is either red or black, but the proportion is unknown. One ball will be drawn from each urn. Empirically, subjects are indifferent between betting on "the ball drawn from urn A is black" and "... red". This also holds for urn B. However, subjects prefer bets in which urn A is involved over bets in which $\mathrm{B}$ is involved. This cannot hold under the Bayesian-rational expectations hypothesis. Gilboa and Schmeidler (1989) propose the following solution. "In case of urn B, the subject has too little information to form a prior. Hence, (s)he considers a set of priors as possible." (p. 142; italics provided). ${ }^{3}$

We consider a setting where the agent has little information about the stochastic component of the performance measure. Therefore, the agent's beliefs about the distribution of the shock on the performance measure are not represented by a single probability distribution, but instead by a set of probability distributions. We use Gilboa and Schmeidler's (1989) ambiguity aversion concept in which the individual evaluates an act by the probability distribution that yields the lowest expected utility. In our moral hazard framework, this means that the agent is, loosely speaking, pessimistic about the realization of the shock whenever rewards are stochastic, which is necessary in order to create incentives. Because the principal has to ensure participation of the agent, she has to compensate him for his pessimism. This implies that her costs of providing incentives are relatively high and that her expected payoff is relatively low (compared to the orthodox model which neither considers ambiguity nor ambiguity aversion). Because the costs of implementing even small incentives can be non-negligible, the principal may optimally provide no incentives at all, that is, set a fixed wage. In a benchmark case, consid-

\footnotetext{
${ }^{2}$ Similarly, Offerman et al. (2009, p. 1463) state that "[f] or most uncertain events, no objective probabilities of occurrence are known." See also Ghirardato (1994, p. 3).

${ }^{3}$ In contrast, a Bayesian expected utility maximizer nonetheless has a single probability measure and is neutral to ambiguity; see Savage's (1954) axiomatization and the nontechnical discussion of Gilboa, Postlewaite, and Schmeidler (2008).
} 
ered in Section 3.3, the principal always provides weaker incentives with ambiguity than without. Interestingly, in the more general case, the principal may also provide stronger incentives. This can happen when the marginal implementation costs are lower in some range due to ambiguity.

Considering multiple performance measures, we show that it can be optimal for the principal not to contract on an informative but ambiguous performance measure and instead provide incentives solely via other measures. The reason is that, with ambiguity, the inclusion of a measure into a wage scheme causes costs which are not negligible even when the wage depends on the measure only to a small extent. Hence, the Informativeness Principle does not hold in our model.

Related Literature.- Mukerji (1998) shows that ambiguity might endogenously lead to the incompleteness of contracts. He considers a hold-up problem and incompleteness in his setting means that the contract does not include any instruction for some possible events.

In contrast to the finance literature, ${ }^{4}$ the ambiguity concept is rarely used in principal-agent theory. There are a few exceptions. Mukerji (2003) inquires into the impact of ambiguity in procurement contracts under cost uncertainty. His main result is that the optimal contract sets no financial incentives at all to induce exertion of cost-reducing effort when the ambiguity is sufficiently high. ${ }^{5}$ In contrast to our model, only a binary shock realization is considered, the agent is assumed to be risk neutral, and only the case with one performance measure is analyzed. Because of the assumption of risk neutrality, he cannot study the classical tradeoff of the principal-agent literature between providing incentives and sharing risks. Additionally, one cannot discuss the Informativeness Principle when the agent is risk neutral or there is only one performance measure; see the arguments in Section 4. Therefore, we are able to yield many results which cannot be analyzed in Mukerji's (2003) model. We also yield a result which is in contrast to Mukerji (2003). While in his model ambiguity always leads to a lower powered incentive scheme (given that the agent is more averse to ambiguity than the principal), we show that ambiguity may also cause a higher powered scheme.

Kellner (2009) shows in a multiple-agent setting that ambiguity and ambiguity aversion can lead to the superiority of tournaments over wage schemes that only depend on each agent's own output. Ghirardato (1994) and Karni (2009) also belong to this literature, but have different foci than we have: while Ghirardato's main topic is the quality of information,

\footnotetext{
${ }^{4}$ See, for example, Dow and Werlang (1992) or Epstein and Wang (1994).

${ }^{5}$ Lang (2007) studies a model which is similar to Mukerji's (2003), where the agent has to perform two tasks and only the performance of one task can be rewarded.
} 
Karni develops axiomatic foundations of ambiguity aversion.

In Holmström and Milgrom (1991), the total value of the organization cannot be used as a performance measure and the agent has to exert multiple tasks. They show that it can be optimal to set no monetary incentives when only the performance of some tasks can be rewarded (p. 33). We show that also in a setting where the agent exerts a single task it can be optimal not to set incentives. When there are many performance measures, the multiple-task model predicts - like the orthodox model with one task and without ambiguity - that every performance measure which is informative should be included in a compensation scheme, given that positive efforts should be implemented. ${ }^{6}$ This is in contrast to our model where it can be optimal to contract only on a subset of all informative performance measures.

Our model can also be interpreted as a principal-agent setting with noncommon priors; see Section 5.3. In Fang and Moscarini (2005), the principal and the agents have different priors about the agent's abilities. They investigate the implications of worker overconfidence and do not consider ambiguity. ${ }^{7}$

In the next section, we present the model. We analyze it in Section 3. In Section 4, we consider the case with multiple performance measures. In Section 5, we discuss our model and its results. Section 6 concludes.

\section{THE MODEL}

The Moral Hazard Problem. - We use the normal distribution, linear contract, exponential utility structure popularized by Holmström and Milgrom (1987) in the specification of Bolton and Dewatripont (2005, Ch. 4.2). The principal (she) wants to induce the agent (he) to invest effort. Unfortunately, effort is not contractible. Only performance $q=$ $a+\varepsilon$ is contractible, where $a \in \mathbb{R}_{+}$is agent's effort and $\varepsilon$ a random factor/shock. The principal makes a take-it-or-leave-it offer to the agent. It is assumed that the contract can only specify a wage scheme which is linear in performance: $w=t+s q$, where $t$ is the fix component and $s$ is the variable component, where $s$ can be interpreted as the incentive power provided by the contract. When the agent rejects the contract, he receives a monetary payoff of $\bar{w}$ and the principal receives $\bar{\pi}$, where

\footnotetext{
${ }^{6}$ See Feltham and Xie (1994), which show that "[t] he value of an additional measure is zero if, and only if, the existing measures constitute a sufficient statistic for the additional measure with respect to the manager's action." (p. 430).

${ }^{7}$ For other principal-agent models where parties have different priors, see Hvide (2002), de la Rosa (2007), and Santos-Pinto (2008).
} 
$\bar{w}+\bar{\pi}<0 .{ }^{8}$ When the agent accepts, he chooses effort which causes private costs of $\psi(a)=\frac{1}{2} c a^{2}$, where $c>0$ is a cost parameter. ${ }^{9}$ Then the shock realizes and the wage payment is made in accordance with the contract. The principal is risk neutral and his payoff is $q-w$. The agent's utility is $u(\cdot)=-e^{-\eta[w-\psi(a)]}$, where $\eta>0$ is the coefficient of absolute risk aversion. We later clarify which assumptions are crucial for our results and which are not.

Ambiguity. - Let there be a finite number of probability distributions of the shock that are plausible for the agent, given his knowledge. The agent is ambiguity averse in the sense of Gilboa and Schmeidler (1989). The principal is ambiguity neutral and has, for simplicity, the same knowledge as the agent. Therefore, and in contrast to the agent, the principal is able to aggregate all plausible distributions and thereby form a unique prior. We assume that the principal puts a positive (though possibly arbitrary small) weight on each plausible distribution and that her aggregated distribution is normal. We normalize the units in which the shock is measured such that the principal's aggregated distribution is $\varepsilon \sim N\left(0, \sigma^{2}\right) .{ }^{10}$ We further assume that the plausible distributions are stochastically independent. In Section 5.1, we discuss some aspects of the described structure.

Given stochastic independence, and because the principal's aggregated distribution is normal, Cramér's (1936) Theorem implies that the plausible probability distributions are normal, too. The set of plausible distributions is called $\mathcal{G}=\{1, \ldots, g, \ldots, G\}$, with $G \geq 1$. The generic element $g$ is a distribution which is characterized by its mean $\mu_{g}$ and variance $\sigma_{g}^{2}$. We assume that for all plausible distributions $\sigma_{g}^{2}>0$; otherwise we cannot aggregate all plausible distributions to $\varepsilon \sim N\left(0, \sigma^{2}\right)$.

D E F I N I T I O N 1: When $\mathcal{G}$ contains only one element $(G=1)$, there is no ambiguity. When it contains more elements $(G>1)$, there is ambiguity.

So when only one distribution is plausible for the agent, there is no ambiguity. When there are multiple plausible distributions, there is ambiguity.

\footnotetext{
${ }^{8}$ We add this assumption to make sure that the principal hires the agent.

${ }^{9}$ Quadratic costs yield closed-form solutions but are not crucial for our results.

${ }^{10}$ This facilitates comparisons to the orthodox model where it is assumed that there is no ambiguity and all parties evaluate the wage scheme according to $\varepsilon \sim N\left(0, \sigma^{2}\right)$.
} 


\section{ANALYSIS}

\subsection{THE AGENT'S PROBLEM}

The agent has two problems. First, evaluate the principal's offer (and decide whether or not to accept it). Second, to choose effort when he accepted.

Being ambiguity averse in the sense of Gilboa and Schmeidler (1989), the agent assigns to each possible effort and contract the plausible distribution which leads to the lowest expected utility. That is, the agent evaluates a wage scheme according to the, loosely speaking, most pessimistic plausible distribution. Which plausible distribution is most pessimistic depends on the wage scheme.

Calculations similar to the ones in Bolton and Dewatripont (2005) yield that the certainty equivalent of the agent is

$$
\left.\hat{w}(\cdot)\right|_{g}=t+s a+s \mu_{g}-\frac{1}{2} \eta s^{2} \sigma_{g}^{2}-\frac{1}{2} c a^{2} .
$$

Observe that the agent's two problems are separable in the sense that the agent's selection of the optimal effort level is independent from the evaluation of the contract. This simplifies the analysis greatly.

When the agent accepts the principal's offer, he selects the level of effort which maximizes his expected utility or equivalently his certainty equivalent. From the first-order condition of (1) and because the secondorder condition is satisfied, we get that

$$
a^{*}= \begin{cases}s / c & \text { for } s \geq 0 \\ 0 & \text { otherwise }\end{cases}
$$

The agent evaluates the scheme $(s, t)$ according to the plausible distribution $\check{g}$ which minimizes his expected payoff:

$$
\left.\check{g} \in \operatorname{argmin}_{g \in \mathcal{G}} \hat{w}(\cdot)\right|_{g} .
$$

It is possible that $\check{g}$ is not unique. As will become clear below, this causes no problems. Finally note that the agent accepts the principal's offer if and only if $\left.\hat{w}\left(a^{*}\right)\right|_{\check{g}} \geq \bar{w}$.

\subsection{THE PRINCIPAL'S PROBLEM}

The principal evaluates the wage scheme according to the distribution $\varepsilon \sim N\left(0, \sigma^{2}\right)$. She solves the following program:

$$
\max _{s, t} \mathbb{E}[q-w] \quad \text { subject to }
$$


(i) the incentive constraint that the agent chooses the effort level which maximizes his expected payoff: $\left.a^{*} \in \operatorname{argmax}_{a} \hat{w}(a)\right|_{\check{g}}$, and

(ii) the participation constraint which guarantees that the agent accepts the principal's offer: $\left.\hat{w}\left(a^{*}\right)\right|_{\check{g}} \geq \bar{w}$,

(iii) the constraint that the agent evaluates the scheme according to the plausible distribution $\check{g}$, which minimizes $\left.\hat{w}(\cdot)\right|_{g}$.

We now seek to simplify the maximization problem. First, from Section 3.1 we know that we can replace the incentive constraint by (2). Second, in the optimum the participation constraint holds with equality; otherwise the principal can decrease $t$ so that her payoff increases. Hence, the principal's problem (4) can be rewritten as

$$
\max _{s} a+s \mu_{\check{g}}-\eta s^{2} \sigma_{\check{g}}^{2} / 2-a^{2} c / 2-\bar{w} \quad \text { subject to }
$$

(i) (2) and

(ii) $\left.\check{g} \in \operatorname{argmin}_{g \in \mathcal{G}} \hat{w}(\cdot)\right|_{g}$.

We first consider the relaxed problem and then continue with the full problem.

Principal's Relaxed Problem.- Suppose that the agent evaluates a contract according to the plausible distribution $g$. Additionally, we assume that $a=s / c$ instead of (2). Then (5) simplifies to the relaxed problem of

$$
\max _{s} \frac{s}{c}+s \mu_{g}-\eta s^{2} \sigma_{g}^{2} / 2-\frac{s^{2}}{2 c}-\bar{w} .
$$

The interpretation is simple: the first part is the effort induced by the contract; the terms $-\left(s \mu_{g}-\eta s^{2} \sigma_{g}^{2} / 2\right)$ are the combined risk and ambiguity premium which can be decomposed into a risk premium $\eta s^{2} \sigma^{2} / 2$ and an ambiguity premium $-s \mu_{g}+\eta s^{2}\left(\sigma_{g}^{2}-\sigma^{2}\right) / 2$; the term $\frac{s^{2}}{2 c}$ represents the equilibrium effort costs; and $\bar{w}$ is the monetary equivalent of the agent's outside option.

Optimization of (6) yields that the principals sets the following incentives:

$$
\left.s^{*}\right|_{g}=\frac{1+c \mu_{g}}{1+\eta c \sigma_{g}^{2}} .
$$

The principal's expected payoff is derived by evaluating (6) with $s=\left.s^{*}\right|_{g}$.

Without Ambiguity. - When there is no ambiguity, $\mathcal{G}$ contains only one element. Then the only plausible distribution $g$ must be characterized by $\mu_{g}=0$ and $\sigma_{g}^{2}=\sigma^{2}$. From (7) we get that the principal sets the incentives

$$
s_{\text {no ambiguity }}^{*}=\frac{1}{1+\eta c \sigma^{2}},
$$

which are always positive. Without ambiguity the solution of the relaxed problem is the solution of the principal's full problem. Observe that when 
there is no ambiguity the agent's ambiguity aversion does not matter and we get the results of the orthodox model. The same is true when there is ambiguity but the agent is ambiguity neutral. Therefore, ambiguity only matters in connection with ambiguity aversion and vice versa.

Returning to the Principal's Full Problem. - The next lemma says that there is always a plausible distribution which has a non-negative mean.

L E M M A 1: There is a $g \in \mathcal{G}$ for which $\mu_{g} \geq 0$.

Next we prove that the principal does not set a contract where the wage is decreasing in performance.

L E M M 2: The principal never sets negative incentives: $s^{*} \nless 0$.

A rough intuition is that setting negative incentives is never optimal because also no incentives $(s=0)$ implement the same effort $a=0$, but the principal's costs are lower than with negative incentives because the agent is fully insured against random factors.

Because in the optimum $s \geq 0$, we can replace the constraint (2) by $s \geq 0$ and substitute $a=s / c$ into the principal's objective function. Moreover, we know from before that the agent's two problems, namely choosing effort and the distribution with which the scheme is evaluated, are separable. Therefore, (5) can be rewritten as

$$
\max _{s} \frac{s}{c}+s \mu_{\check{g}}-\frac{\eta s^{2} \sigma_{\check{g}}^{2}}{2}-\frac{s^{2}}{2 c}-\bar{w} \quad \text { subject to }
$$

(i) $s \geq 0$ and

(ii) $\check{g} \in \operatorname{argmin} s \mu_{g}-\frac{\eta s^{2} \sigma_{g}^{2}}{2}$.

We next seek to answer the question whether or not the principal optimally provides positive incentives.

P R O P O S I T I O N 1: The principal contracts on the performance measure if and only if $\mu_{\underline{g}}>-1 / c$, where $\underline{g} \in \operatorname{argmin}_{g \in \mathcal{G}} \mu_{g}$.

So when there is a plausible distribution with a sufficiently low mean the principal specifies a fixed wage scheme $\left(s^{*}=0\right)$ and sets no incentives at all. Intuitively, due to ambiguity the marginal costs of providing incentives are not negligible even for small incentives whenever $\mu_{g}<0$. Formally, $\lim _{s \rightarrow 0} d($ ambiguity premium $) / d s=-\mu_{g}$. At the same time the marginal benefit of setting small incentives (via inducing a higher 
effort) is $1 / c$. Hence, whenever it is sufficiently costly to provide even small incentives, the principal sets no incentives at all. ${ }^{11}$

The result that the principal may optimally set a fixed wage scheme is in stark contrast to the results of the orthodox model. In a model without ambiguity/ambiguity aversion, there is no ambiguity premium. Then, the principal's marginal costs of providing small incentives are negligible. Therefore the principal always sets incentives, see (8). More technically, when the agent is risk averse but ambiguity neutral, the agent is firstorder neutral towards stochastic payments: $\lim _{s \rightarrow 0} d($ risk premium $) / d s=$ 0 .

Consider next the case where $1+c \mu_{g}>0$. Then the principal will set positive incentives and the first constraint of the principal's problem (9) does not bind. Hence, the principal's problem can be rewritten as

$$
\max _{s} \frac{s}{c}+\min _{g}\left\{s \mu_{g}-\frac{\eta s^{2} \sigma_{g}^{2}}{2}\right\}-\frac{s^{2}}{2 c}-\bar{w} .
$$

The formula can be interpreted as effort minus implementation costs (which in turn consists of the risk and the ambiguity premium, the effort costs, and the agent's outside option). Figure 1 shows how one constructs the principal's implementation costs, given two plausible distributions. A dotted line depicts the implementation costs when the agent uses a particular distribution to evaluate the wage scheme. Due to ambiguity aversion à la Gilboa and Schmeidler (1989), the distribution according to which the agent evaluates the wage scheme is always the one which leads to the higher implementation costs. The bold line shows the principal's actual implementation costs and is constructed out of the segments of both dotted lines. In the right diagram of Figure 1, we added a straight line $s / c$. This is the agent's effort, or equivalently, the principal's benefit from setting the incentives $s$. One can easily solve for the optimal incentives $s^{*}$.

This simple example already clarifies that the implementation costs are always convex. This implies that the optimal contract is unique. Observe that the optimum can easily be characterized for a concrete set of plausible distributions. This is, however, not the case for a general set. One of the difficulties is that one may get corner solutions; see the right diagram of Figure 1. Technically, $s^{*}$ does not necessarily coincide with $\left.s^{*}\right|_{\check{g}}$ from (7). In Section 3.3, we consider a special set of plausible distributions which allows us to yield the solution explicitly.

\footnotetext{
${ }^{11}$ When no incentives are set, zero effort is implemented. It may be more plausible to assume that the agent wants to spend some effort even when no incentives are set. This is indeed the case if the effort cost function satisfies $\psi^{\prime}(a) \leq 0$ for $a \in[0, \bar{a}]$, with $\bar{a}>0$, see Holmström and Milgrom (1991, pp. 33-34).
} 

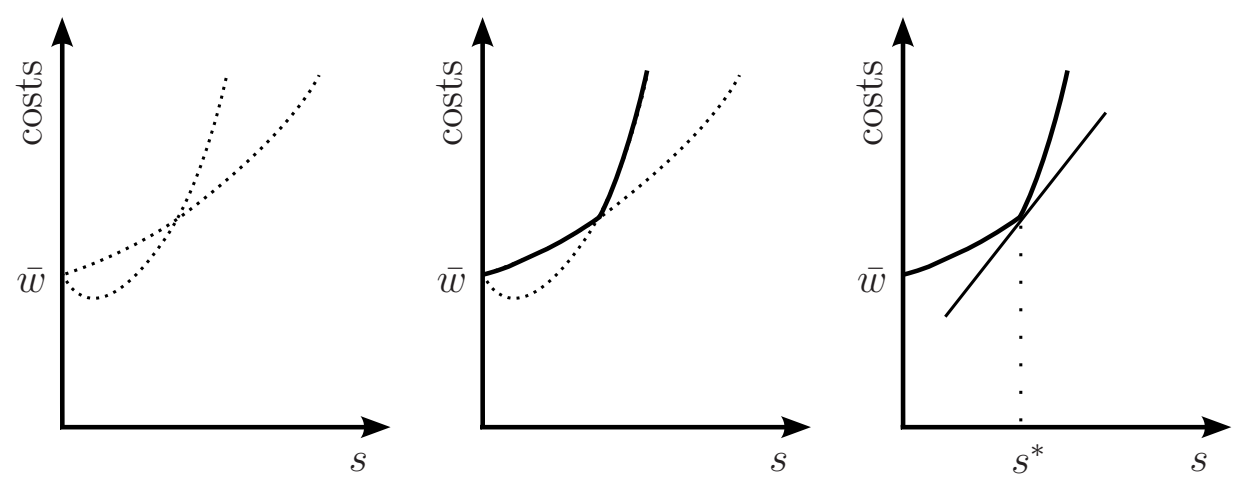

Figure 1: The costs of setting incentives (left, middle) and the optimal incentives (right).

From Figure 1 or (10) it is apparent that $\mu_{\check{g}}$ and $\sigma_{\breve{g}}^{2}$ are weakly increasing in the incentives $s$. For low-powered incentives, the agent evaluates the scheme according to the plausible distribution with the lowest mean. For higher-powered incentives, the agent uses a distribution with a higher mean and variance. Intuitively, because of the agent's risk aversion, highpowered incentive schemes are especially harmful for the agent when the variance of the shock on the performance measure is high. This is not the case for lower-powered schemes because then the agent does not care much about variations.

Can we compare the implementation costs which the principal faces when the agent is ambiguity averse versus when he is not?

P R O P O I T I O N 2: The principal has higher costs of implementing any effort $a>0$ with ambiguity than without. She is strictly worse off with ambiguity than without.

Intuitively, with ambiguity the principal has to compensate the agent not only for his risk premium, but also for his ambiguity premium. This increases the principal's cost of implementing any level of effort, and hence also the principal's cost when the optimal effort is implemented. Observe that a higher degree of risk aversion $\eta$ leads to steeper implementation costs. Hence, the principal sets weakly lower incentives.

Does the principal provide weaker incentives with ambiguity than without? In Section 3.3, we show that this is indeed the case when the plausible distributions can be ordered in a grid; see Proposition 3. This is, however, not true in general. We next provide an example where the principal optimally sets stronger incentives due to ambiguity. The key insight is that stronger incentives can be optimal when the marginal implementation costs are lower for some range with ambiguity than without. 
An Example where Ambiguity Cause Higher-Powered Incentives.Suppose that $\sigma^{2}=\eta=c=1$. Then, without ambiguity, the principal optimally sets

$$
s_{\text {no ambiguity }}^{*}=1 / 2 \text {, }
$$

see (8).

Consider next the case where there is ambiguity and there are two plausible distributions $(G=2)$. The plausible distribution 1 has the following properties: $\mu_{1}=-1 / 6$ and $\sigma_{1}^{2}=1 / 2$. The principal puts a weight of $p_{1} \rightarrow 0$ on the plausible distribution 1 and a weight $p_{2}=$ $1-p_{2}$ on the plausible distribution 2 . Because the principal aggregates all plausible distributions to $\varepsilon \sim N\left(0, \sigma^{2}\right)$, we have $\mu_{2} \approx 0, \sigma_{2}^{2} \approx 1$.

From (1) and (3) we get that the agent evaluates the wage scheme according to the plausible distribution 1 when $0 \leq s \lesssim 2 / 3$. From (7) we get that the principal optimally sets $\left.s^{*}\right|_{1}=10 / 18$, given that the agent evaluates the wage scheme according to plausible distribution 1 . Because $\left.s^{*}\right|_{1}=10 / 18$ is below $2 / 3$, the agent indeed uses the plausible distribution 1 . Taking as given that the agent evaluates the wage scheme according to the plausible distribution 2 , the principal optimally sets $\left.s^{*}\right|_{2}$ which is approximately $1 / 2$. But for $s \approx 1 / 2$ the agent evaluates the wage scheme according to the plausible distribution 1 and not 2 . It is straightforward to show that setting incentives so that the agent is indifferent between both plausible distributions is worse for the principal than setting $\left.s^{*}\right|_{1}=10 / 18$. Hence, with ambiguity the principal optimally sets incentives of

$$
s_{\text {ambiguity }}^{*}=10 / 18,
$$

which is above the incentives set without ambiguity.

The intuition is that the plausible distribution 1 has a low mean $\mu_{1}$ which causes the absolute implementation costs to be higher with ambiguity than without (or with the plausible distribution 2, at least for incentives up to $s \approx 2 / 3$ ). But due to the low variance $\sigma_{1}^{2}$, the marginal implementation costs for incentives of $s \approx 1 / 2$ are lower with ambiguity than without. Hence, the principal optimally sets higher-powered incentives with ambiguity than without.

\subsection{PLAUSIBLE DISTRIBUTIONS IN A GRID}

Suppose that there are $M$ means and $N$ variances which are plausible for the agent. All combinations are plausible, too, resulting in $M \times N$ plausible distributions which can be ordered in a grid. See Figure 2 for a $4 \times 3$ example. The set of parameters characterizing the plausible distributions is

$$
\mathcal{S}:=\left\{\left(\mu_{m}, \sigma_{n}^{2}\right) \mid \mu_{m} \in \boldsymbol{\mu}, \sigma_{n}^{2} \in \boldsymbol{\sigma}^{\mathbf{2}}\right\}
$$




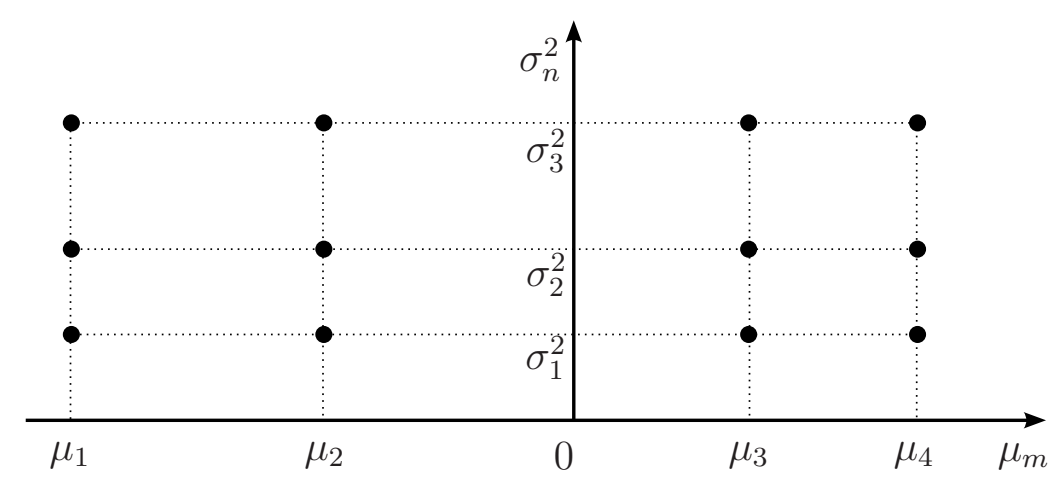

Figure 2: A $4 \times 3$ example of plausible distributions.

with $\boldsymbol{\mu}:=\left(\mu_{1}, \ldots, \mu_{m}, \ldots, \mu_{M}\right)$ and $\boldsymbol{\sigma}^{\mathbf{2}}:=\left(\sigma_{1}^{2}, \ldots, \sigma_{n}^{2}, \ldots, \sigma_{N}^{2}\right)$, where we assign the numbers such that $\mu_{m}<\mu_{m+1}$ and $\sigma_{n}^{2}<\sigma_{n+1}^{2}$.

The following definition is useful.

D E F I N I T I O 2: There is more ambiguity when $\mu_{1}$ decreases and $\sigma_{N}^{2}$ does not decrease, or $\mu_{1}$ does not increase and $\sigma_{N}^{2}$ increases.

The motivation is as follows: when the agent misses more information regarding the probabilities, the set of plausible distributions $\left(\boldsymbol{\mu}\right.$ and $\boldsymbol{\sigma}^{\mathbf{2}}$ ) is more dispersed which results into a decrease of $\mu_{1}$ and an increase of $\sigma_{N}^{2}{ }^{12}$ Observe that for any contract which positively depends on the performance measure, an ambiguity-averse agent is worse off when there is more ambiguity in the sense of Definition 2.

L E M M A 3: Suppose $M \times N>1$. Then $\mu_{1} \leq 0$ and $\sigma_{N}^{2}>\sigma^{2}$. The first inequality is strict for $M \geq 2$.

In words, the plausible distribution with the lowest mean has a mean which is smaller or equal to the mean of the principal's aggregated distribution, and the plausible distribution with the highest variance has a variance which is greater than the variance of the principal's aggregated distribution. ${ }^{13}$

Because the plausible distributions can be ordered in a grid it is very simple to determine the distribution according to which the agent evaluates a contract. When the principal sets a contract with positive incentives $s>0$, then the agent's lowest expected utility is yielded when

\footnotetext{
${ }^{12}$ One can think of the wider dispersion as a mean preserving spread of $\boldsymbol{\mu}$ and $\boldsymbol{\sigma}^{2}$.

${ }^{13}$ It is easy to show that Lemma 3 is also true for the case in which the realizations of the plausible distributions are correlated (and the plausible distributions are still normal). There is one exception: when the correlation is 1 and $N=1$, we have $\sigma^{2}=\sigma_{N}^{2}$.
} 
the variance is maximal and the mean minimal. So the agent evaluates a wage scheme with $s>0$ according to the plausible distribution $[1, N]$.

When the principal provides a contract without incentives $s=0$ and all plausible distributions yield the same expected utility. Without loss of generality we can hence assume that also in this case the agent uses the plausible distribution $[1, N]$. Because of Lemma 2 , we do not have to consider cases with negative incentives $s<0$.

Using the previous insights, the principal's maximization problem simplifies to

$$
\max _{s} \frac{s}{c}+s \mu_{1}-\eta s^{2} \sigma_{N}^{2} / 2-\frac{s^{2}}{2 c}-\bar{w} \text { s.t. } s \geq 0 .
$$

Optimization yields that the principals sets the following incentives:

$$
s^{*}= \begin{cases}\frac{1+c \mu_{1}}{1+\eta c \sigma_{N}^{2}} & \text { for } 1+c \mu_{1}>0 \\ 0 & \text { otherwise }\end{cases}
$$

Proposition 1 also applies here: the principal sets no incentives if and only if $\mu_{1} \leq-1 / c$. From Lemma 3 we know that $\mu_{1} \leq 0$ and $\sigma_{N}^{2}>\sigma^{2}$. Hence, we have an additional result.

P R O P O S I T I O N 3: Suppose that the plausible distributions can be ordered in a grid. With ambiguity the incentives set by the principal are weaker than without ambiguity.

The intuition is that when the plausible distributions can be ordered in a grid, the marginal implementation costs are always higher with ambiguity than without.

Plugging (12) into (11) yields the principal's expected payoff in equilibrium:

$$
\mathbb{E}[q-w]^{*}= \begin{cases}\frac{\left(1+c \mu_{1}\right)^{2}}{2 c\left(1+\eta c \sigma_{N}^{2}\right)}-\bar{w} & \text { for } 1+c \mu_{1}>0 \\ -\bar{w} & \text { otherwise. }\end{cases}
$$

So the principal is worse off with ambiguity than without; see also Proposition 2 .

An advantage when the plausible distributions are ordered in a grid is that one can yield closed-form solutions and therefore directly gets the comparative statics.

Comparative Statics. - First, more ambiguity leads to the provision of weaker incentives: $d s^{*} / d \mu_{1}>0$ and $d s^{*} / d \sigma_{N}^{2}<0$. Intuitively, more ambiguity increases the marginal costs of providing incentives. Hence, the principal sets weaker incentives. 
Second, more ambiguity leads to a lower expected payoff for the principal: $d \mathbb{E}[q-w]^{*} / d \mu_{1} \geq 0$ and $d \mathbb{E}[q-w]^{*} / d \sigma_{N}^{2} \leq 0$, where for $s^{*}>0$ the inequalities are strict. The logic is simple: the principal can set the same incentives with little ambiguity than with a lot of ambiguity. Because more ambiguity leads to higher costs for the principal in order to provide a certain level of incentives (see arguments before), the principal must be better off with little ambiguity.

Third, in the orthodox model as well as in the model with ambiguity a higher degree of risk aversion $\eta$ (at least weakly) decreases the optimal incentives and the principal's payoff.

\section{ADDITIONAL INFORMATION: VIOLATION OF THE INFOR- MATIVENESS PRINCIPLE}

We start with two remarks. First, when no effort is implemented, there is no need to use any performance measure in the wage scheme. ${ }^{14}$ Hence, it is only useful to interpret the Informativeness Principle in the way that whenever some effort is implemented, any measure of performance that reveals information about effort is included in the contract. Trivially, when there is just one measure, this measure must be used; otherwise no incentives are set. We therefore now consider the interesting case where there are multiple measures of performance. Second, the agent's risk aversion is crucial to discuss the Informativeness Principle. When the agent is risk neutral, the first-best is implementable even with one (nonambiguous) performance measure. Then the principal does not care whether there is another performance measure or not.

Suppose there are two performance measures $Y$ and $Z$ with realizations $y$ and $z$, respectively. Similar to the case with one performance measure, we assume that

$$
y=a+\varepsilon_{Y}, z=a+\varepsilon_{Z},
$$

and that the principal's expected payoff is $a-\mathbb{E}[w]$. We initially assume that $\varepsilon_{Y}$ and $\varepsilon_{Z}$ are uncorrelated. Moreover, we assume that the agent only feels ambiguity with respect to the performance measure $Z$ and he is again ambiguity averse. The contract takes the form $w=t+s_{Y} y+s_{Z} z$. The principal evaluates the wage scheme according to $\varepsilon_{Y} \sim N\left(0, \sigma_{Y}^{2}\right)$ and $\varepsilon_{Z} \sim N\left(0, \sigma_{Z}^{2}\right)$. The agent uses $\varepsilon_{Y} \sim N\left(0, \sigma_{Y}^{2}\right)$ to evaluate a wage scheme. On the measure $Z$ we impose the same assumptions as in Section 3.3. That is, we consider the case where the plausible distributions can be

\footnotetext{
${ }^{14}$ With linear schemes no effort can be implemented when the wage does not depend, or negatively depends, on the performance measure(s).
} 
ordered in a grid. ${ }^{15}$ So

$$
\mathcal{S}_{\mathcal{Z}}:=\left\{\left(\mu_{Z, m}, \sigma_{Z, n}^{2}\right) \mid \mu_{Z, m} \in \boldsymbol{\mu}_{Z}, \sigma_{Z, n}^{2} \in \boldsymbol{\sigma}_{Z}^{2}\right\},
$$

with $\boldsymbol{\mu}_{\boldsymbol{Z}}:=\left(\mu_{Z, 1}, \ldots, \mu_{Z, m}, \ldots, \mu_{Z, M}\right)$ and $\boldsymbol{\sigma}_{\boldsymbol{Z}}^{2}:=\left(\sigma_{Z, 1}^{2}, \ldots, \sigma_{Z, n}^{2}, \ldots, \sigma_{Z, N}^{2}\right)$, where we assign the numbers such that $\mu_{Z, m}<\mu_{Z, m+1}$ and $\sigma_{Z, n}^{2}<\sigma_{Z, n+1}^{2}$.

The next proposition states that it is not always optimal for the principal to include both performance measures into the contract. ${ }^{16}$

P R O P O S I I O N 4: The principal always contracts on the performance measure $Y$. She contracts on the performance measure $Z$ if and only if

$$
\mu_{Z, 1}>-\frac{1}{c+1 /\left(\eta \sigma_{Y}^{2}\right)}
$$

In the orthodox model (without ambiguity/ambiguity aversion), it is always optimal to use both measures (see Holmström 1979). The intuition is as follows. Suppose the optimal contract depends only on the measure $Y$. By making the contract slightly dependent on the measure $Z$ the agent's risk premium does not increase marginally(keeping the effort level fix), because the agent is locally risk neutral. However, the agent's incentives are improved. This allows the principal to make the contract less dependent on $Y$ (so that the agent chooses the same effort level), which decreases the agent's risk premium and thereby also the principal's costs. Hence, the optimal contract uses $Y$ and $Z$.

This arguments do not hold with ambiguity. From Section 3.2 we know that the costs of making a contract even slightly dependent on an ambiguous performance measure are non-negligible whenever the agent is averse to ambiguity. Therefore, when there is a lot of ambiguity (i.e., when $\mu_{Z, 1}$ is low) the principal does not use the measure $Z$, even though $Z$ reveals information. This finding is valuable because "the Informativeness Principle, i.e., that all factors correlated with performance should be included in a compensation contract, seems to be violated in many occupations" (Prendergast 1999, p. 21).

\footnotetext{
${ }^{15}$ The results directly carry over to the more general case where this need not be true. One simply has to replace $\mu_{Z, 1}$ with $\mu_{\underline{g}}$ in the formulas below, where $\underline{g}_{Z}$ is the plausible distribution of $Z$ which has the lowest mean. The idea is the same as in the proof of Proposition 1. The principal's implementation costs when one fixes the distribution which the agent uses to evaluate the wage scheme to $\underline{g}_{Z}$ are weakly lower than the actual implementation costs. When the ambiguous measure $Z$ is used only to a small extent, then the agent indeed uses the distribution $\underline{g}_{Z}$ to evaluate the wage scheme. Therefore, when we want to determine whether or not the principal uses the ambiguous performance measure $Z$ we can restrict the attention to distribution $g_{Z}$.

${ }^{16}$ When the agent feels ambiguity with respect to both measures, the principal may optimally neglect both measures; that is, set a fixed wage.
} 
Observe that the condition that the ambiguous measure is used is stronger when there is another nonambiguous measure (this is the case considered in this section; see Proposition 4) than when there is no other measure (Section 3; see Proposition 1), because

$$
-\frac{1}{c+1 /\left(\eta \sigma_{Y}^{2}\right)}>-\frac{1}{c}
$$

The reason is simple. When there is another measure, the principal can still provide incentives when she uses the other measure. This type of substitution is not possible when there is no other measure. Because the agent's marginal effort costs are the lowest for small incentives, it is attractive for the principal to set at least some incentives. Therefore, without another measure the principal is more eager to contract on the ambiguous measure. Note that when $\sigma_{Y}^{2} \rightarrow \infty$, the measure $Y$ is not useful for the principal because the variance is huge. Then the thresholds in (14) are the same in the limit.

The next proposition states that the principal may also not contract on the measure $Z$ when $\varepsilon_{Y}$ and $\varepsilon_{Z}$ are correlated, where $\rho$ is the correlation coefficient.

P R O P OSITION 5: When $\rho<0$, the principal contracts on the performance measure $Z$ if and only if

$$
\mu_{Z, 1}>\frac{\eta \sigma_{Y}\left(\rho \sigma_{Z, 1}-\sigma_{Y}\right)}{1+\eta c \sigma_{Y}^{2}}
$$

When $\rho>0$, she contracts on the measure $Z$ if and only if

$$
\mu_{Z, 1}>\frac{\eta \sigma_{Y}\left(\rho \sigma_{Z, N}-\sigma_{Y}\right)}{1+\eta c \sigma_{Y}^{2}} \text { or } \mu_{Z, M}<\frac{\eta \sigma_{Y}\left(\rho \sigma_{Z, 1}-\sigma_{Y}\right)}{1+\eta c \sigma_{Y}^{2}}
$$

\section{DISCUSSION}

\subsection{PRINCIPAL'S AGGREGATION PROBLEM AND THE EFFECTS OF AN AMBIGUITY-AVERSE PRINCIPAL}

We assumed that aggregating all the distributions which are plausible for the agent yields the principal's aggregated distribution $\varepsilon \sim N\left(0, \sigma^{2}\right)$. What happens when one departs from this assumption? As long as the principal aggregates to some distribution, we can still normalize the shock such that the principal evaluates the wage scheme according to the distribution $\varepsilon \sim N\left(0, \sigma^{2}\right)$. Then the problem does not change qualitatively, at least when there exists a plausible normalized distribution for the agent with a non-negative mean. 
If this is not the case, so that all plausible distributions have negative means, the principal is better off with negative than with zero incentives. It may turn out that the optimal negative incentives lead to a higher expected payoff for the principal than the optimal positive incentives. That is, the principal may optimally set negative incentives and Lemma 2 therefore no longer holds. ${ }^{17}$

Suppose next that also the principal is ambiguity averse and that she has the same set of plausible priors as the agent. For small positive incentives, the worst distribution for the principal is the one with the highest mean, while for the agent the worst distribution is the one with the lowest mean. Obviously, the principal's ambiguity aversion makes it more likely that she optimally sets no incentives at all when there is one performance measure, or does not use an ambiguous measure when several measures are available.

\subsection{ROBUSTNESS AND ALTERNATIVE CONCEPTS OF AMBIGUITY AVERSION}

The feature of Gilboa and Schmeidler's (1989) model that the agent assigns to each wage scheme the lowest expected value over his set of priors is not important for our results. Following Ghirardato, Maccheroni, and Marinacci (2004), suppose instead that the agent's preferences can be represented by

$$
\left.\alpha \min _{g \in \mathcal{G}} \hat{w}(\cdot)\right|_{g}+\left.(1-\alpha) \max _{g \in \mathcal{G}} \hat{w}(\cdot)\right|_{g}
$$

with $\alpha \in[0,1]$. Clearly, when $1+c \mu_{\underline{g}}<0$, where $\underline{g} \in \operatorname{argmin}_{g \in \mathcal{G}} \mu_{g}$, and $\alpha$ is sufficiently close to 1 , the principal sets no incentives when there is one performance measure. Our results regarding multiple performance measures are robust in the same sense.

It is crucial for our results that the ambiguity-averse agent is not ambiguity neutral in the limit when gambles become small. When the agent is ambiguity neutral in the limit, then making the wage scheme dependent on an ambiguous performance measure has only a second-order effect on the agent's ambiguity premium and thereby on the principal's implementation costs. In Klibanoff, Marinacci, and Mukerji's (2005) concept of smooth ambiguity aversion, the individual is ambiguity neutral

\footnotetext{
${ }^{17}$ Then the principal and the agent are essentially betting. The betting motive is then so strong that it dominates the motive to implement positive effort. See also Billot et al. (2000). They show that agents with noncommon priors and preferences à la Gilboa and Schmeidler (1989) take bets when they have not at least one common prior.
} 
in the limit when the function $\phi$ is strictly increasing, continuous, and differentiable.

Maccheroni, Marinacci, and Rustichini (2006) weaken the independence axiom considered by Gilboa and Schmeidler (1989). Therefore, they yield a generalization of their model. Because the multiple prior model remains as a special case, we can be sure that our results also hold in some specifications of their model. However, as the authors argue, "the limit behavior of variational preferences is described by multiple priors preferences, but the size of the sets of priors they feature is very different" (p. 1459). For some classes of variational preferences, this set is just a singleton, so that the limit preference is just the subjective expected utility preference and the individual is ambiguity neutral in the limit. ${ }^{18}$

The normal distribution, linear contract, exponential utility structure is not very general. ${ }^{19}$ An obvious question is whether or not our results can be expected to hold in a more general model. We have the following conjecture. Also in a more general model it should hold that the ambiguity-averse agent evaluates a wage scheme pessimistically. Therefore, when the principal contracts on an ambiguous performance measure the compensation demanded by the agent is relatively high, compared to the orthodox model. This implies that the principal's costs of providing incentives are relatively high and that she yields a relatively low expected payoff. Additionally, unless the agent is ambiguity neutral in the limit, the compensation he demands is not negligible even for small incentives. This implies that the principal may optimally not contract on an ambiguous performance measure.

In more abstract terms, we think that the kernel of the Ellsberg (1961) paradox directly predicts that the principal may optimally not contract on an ambiguous performance measure. Contracting on an ambiguous performance measure is like contracting on an urn with unknown probabilities. The principal has to provide a compensation to the agent when contracting on an ambiguous performance measure. When there is a lot of ambiguity, the agent demands a compensation which may be so high that the principal optimally does not contract on the ambiguous measure.

\subsection{WHEN PARTIES "AGREE TO DISAGREE"}

The model stays mathematically equivalent when there is neither ambiguity nor ambiguity aversion, but the agent and the principal have different

\footnotetext{
${ }^{18}$ Maccheroni, Marinacci, and Rustichini (2006) call this second-order ambiguity sensitivity; see especially p. 1873.

${ }^{19}$ Linear contracts are optimal in the dynamic setting of Holmström and Milgrom (1987). It is an open question whether this result holds with ambiguity.
} 
opinions about the distribution of the shock. That is, both parties "agree to disagree". Suppose that the principal thinks that the shock is normally distributed with variance $\sigma^{2}$ and a (normalized) mean of 0 , whereas the agent thinks that the shock is normally distributed with variance $\sigma_{N}^{2}$ and mean $\mu_{1}$. When the agent is more pessimistic about the distribution than the principal we have $\mu_{1}<0$ and $\sigma_{N}^{2}>\sigma^{2}$. Observe hat this is just the case we have considered before when we have ambiguity/ambiguity aversion and the plausible distributions which can be ordered in a grid. The agent may also be more optimistic than the principal so that we have $\mu_{1}>0$ and $\sigma_{N}^{2}<\sigma^{2}$. Then the principal sets very high-powered incentives and receives a very high perceived expected payoff, compared to the case with a common prior.

\subsection{AGENT'S WELFARE}

Suppose the agent is either ambiguity averse or not. Given that there is ambiguity, when is he better off from an ex post perspective? In both cases, the principal specifies the offer such that the agent's perceived expected utility equals the utility from his reservation payoff $\bar{w}$. However, when the agent is ambiguity averse, the actual expected utility ${ }^{20}$ exceeds the perceived expected utility. Hence, from an ex post perspective the agent obtains a higher surplus with ambiguity aversion than without it. The agent's higher surplus is at the principal's expense; see Proposition 2.21

Do these results also hold with multiple principals? Suppose multiple principals compete perfectly for the agent. Then, in equilibrium all principals must make zero expected profits. This is true with or without ambiguity aversion of the agent. Hence, the principals' profits do not change due to agent's ambiguity aversion. The expected total surplus is $\mathbb{E}[q-w]+\tilde{w}$, where $\tilde{w}=t+s a-\frac{1}{2} \eta s^{2} \sigma^{2}-\frac{1}{2} c a^{2}$ is the actual certainty equivalent. Maximization of the expected total surplus yields that $s_{\text {total }}^{*}=1 /\left(1+\eta c \sigma^{2}\right)$. Observe that this is the level of incentives provided when there is no ambiguity aversion. With ambiguity aversion, the principal sets generically different incentives. Hence, with an ambiguityaverse agent, the expected total surplus is lower than without ambiguity aversion. From before we know that with perfect competition the principals' profits do not change due to agent's ambiguity aversion. Hence, from an ex post perspective the agent is worse off with ambiguity aversion

\footnotetext{
${ }^{20}$ To evaluate welfare, we assume that the principal's prior is correct.

${ }^{21}$ That a party profits from its distorted prior is non-standard. For example, in DellaVigna and Malmendier (2004), the party with the undistorted prior exploits the party with the distorted prior.
} 
than without it. This result is the reverse yielded with only one principal. Therefore, whether or not the agent is better off with ambiguity aversion crucially depends on the competitiveness of the environment.

\subsection{AMBIGUITY ABOUT PRODUCTIVITY}

We have assumed that the agent's knowledge about the shock $\varepsilon$ is ambiguous. What can we say when the ambiguity concerns the productivity of effort? To formalize this, suppose that the agent takes several productivity parameters $\beta_{j}$ as plausible. The agent evaluates the wage scheme according to

$$
q=\beta_{j} a+\varepsilon,
$$

where $\beta_{j} \in\left\{\beta_{1}, \ldots, \beta_{J}\right\}$, with $\beta_{j}<\beta_{j+1}$. We assume that $0<\beta_{1}<1<$ $\beta_{J}$. Note that the principal evaluates the contract still with $q=a+\varepsilon$.

We look at the principal's subproblem of determining the cost minimizing contract for the desired effort level. Recognize that cost minimization is a necessary condition for the optimality of the principal's contract. When the principal wants to implement the effort level $a=0$, then ambiguity concerning the productivity of effort has no effect at all.

There is, however, an effect when the principal wants to implement positive effort $a>0$. Then the principal has to provide positive incentives $s>0$ and the $\beta_{j}$ which leads to lowest expected payoff for the agent is $\beta_{1}$. That is, the agent evaluates the wage scheme according to the productivity parameter $\beta_{1}$.

One can rewrite (15) as

$$
q=a+\dot{\varepsilon}_{a}
$$

when one defines $\dot{\varepsilon}_{a}:=\varepsilon-\left(1-\beta_{1}\right) a$. This transformation allows us to use the insights we have yielded in the main part of the paper. Ambiguity concerning the productivity of effort has the following effect: it negatively shifts the mean of the plausible distribution with which the agent evaluates the wage scheme whenever a positive level of effort should be implemented. Therefore, ambiguity concerning effort (i) increases the principal's costs of implementing any positive effort level; (ii) weakly decreases the implemented effort level; and (iii) makes it possible that the principal does not provide any incentives at all.

\section{CONCLUSIONS}

We considered a principal-agent relationship with moral hazard where the agent's knowledge about the performance measure is ambiguous and he is 
averse to ambiguity. When the principal contracts on the ambiguous performance measure the compensation demanded by the agent is relatively high, compared to the orthodox model (without ambiguity/ambiguity aversion). This implies that the principal's costs of providing incentives are relatively high and that she yields a relatively low expected payoff. Because the compensation demanded by the agent is not negligible even for small incentives, the principal may optimally provide no incentives at all. Moreover, the famous Informativeness Principle does not hold: it can be optimal for the principal to ignore an informative but ambiguous performance measure and instead provide incentives solely via other measures.

Due to ambiguity the agent is pessimistic about the shock on the performance measure. Because this comes at the costs of the principal (via the ambiguity premium demanded by the agent) she may try to convince the agent that there is no reason for pessimism. One way to do this is to provide information to the agent. A problem might be that the principal may have the opportunity to act strategically and provide only information about the shock which indicates that high realizations are very likely. Therefore, it may not be credible that the principal provides information which reduce the agent's ambiguity.

Rustichini (2005, p. 1625) shows that "[p] eople slowly adjust to ambiguity" because "the ambiguity premium declines as subjects repeat their choices". Therefore, an empirical prediction of our model is that longserving performance measures have a comparative advantage over new measures. Hence, the use of performance measures should be history dependent and long-used measures should have some persistence.

\section{APPENDIX}

\subsection{PROOF OF LEMMA 1}

We prove by contradiction. We assumed that aggregating the plausible distributions yields the principal's aggregated distribution which is $\varepsilon \sim$ $N\left(0, \sigma^{2}\right)$. Hence,

$$
\sum_{\mathcal{S}} p_{g} \mu_{g} \stackrel{!}{=} 0
$$

where $p_{g}$ is the probability assigned by the principal that the shock is drawn from the plausible distribution $g$. When $\mu_{g}<0$ for all $g \in \mathcal{G}$ then $\sum_{\mathcal{S}} p_{g} \mu_{g}>0$, which cannot hold because of (17). 


\subsection{PROOF OF LEMMA 2}

Suppose, in contradiction, that the principal sets negative incentives so that $s^{*}<0$. Then the agent chooses $a=0$, see (2). The principal's payoff is then

$$
s^{*} \mu_{g}-\eta\left(s^{*}\right)^{2} \sigma_{g}^{2} / 2-\bar{w} .
$$

The agent evaluates the scheme $(s, t)$ according to the plausible distribution

$$
\check{g} \in \operatorname{argmin}_{g \in \mathcal{G}} s^{*} \mu_{g}-\eta\left(s^{*}\right)^{2} \sigma_{g}^{2} / 2 .
$$

Because there exists a $g$ for which $\mu_{g} \geq 0$, see Lemma 1 , and $\sigma_{g}^{2}>0$ we have

$$
s^{*} \mu_{\check{g}}-\eta\left(s^{*}\right)^{2} \sigma_{\breve{g}}^{2} / 2<0 .
$$

Hence, the principal's expected payoff is below $-\bar{w}$. With $s=0$ it would be $-\bar{w}$. Hence, setting $s^{*}<0$ cannot be optimal.

\subsection{PROOF OF PROPOSITION 1}

First we prove the "if" part. Suppose that the principal solves the program (9) with $\check{g}=\underline{g}$ instead of constraint (ii). Then the problem modifies to

$$
\max _{s} \frac{s}{c}+s \mu_{\underline{g}}-\frac{\eta s^{2} \sigma_{\underline{g}}^{2}}{2}-\frac{s^{2}}{2 c}-\bar{w} \quad \text { subject to } s \geq 0 .
$$

Ignoring the constraint, the derivative is

$$
\frac{d \cdots}{d s}=1 / c+\mu_{\underline{g}}-\eta s \sigma_{\underline{g}}^{2}-s / c
$$

When this derivative is non-positive at $s=0$ then the principal optimally sets $s=0$ to maximize (19). This is equivalent to the condition

$$
1+c \mu_{\underline{g}} \leq 0
$$

Note that for $s=0$ the values of (9) and (19) are identical and that for all $s>0$ (9) is weakly higher then (19). Hence, when the principal optimally sets $s=0$ to maximize (9), then $s=0$ also maximizes (19). Therefore, $s^{*}=0$.

Finally we prove the "only if" part. When $s \rightarrow 0$ then $\check{g}=\underline{g}$ because $\check{g} \in \operatorname{argmin} s \mu_{g}-\frac{\eta s^{2} \sigma_{g}^{2}}{2}$. Therefore, solving (9) for $\check{g}=\underline{g}$ yields that $s^{*}=0$ is optimal only if $1+c \mu_{\underline{g}} \leq 0$. 


\subsection{PROOF OF PROPOSITION 2}

We first show that because of ambiguity the principal faces higher costs of implementing any $a>0$. By (10), implementing an effort $a>0$ requires that $s>0$. We henceforth suppose that $s>0$.

Because of stochastic independence of the plausible distributions, we have that

$$
\sum_{\mathcal{G}} p_{g} \varepsilon_{g} \sim N\left(\sum_{\mathcal{G}} p_{g} \mu_{g}, \sum_{\mathcal{G}} p_{g}^{2} \sigma_{g}^{2}\right) .
$$

The assumption that the principal aggregates the priors to $\varepsilon \sim N\left(0, \sigma^{2}\right)$ requires that

$$
\sum_{\mathcal{G}} p_{g} \mu_{g} \stackrel{!}{=} 0 \text { and } \sum_{\mathcal{G}} p_{g}^{2} \sigma_{g}^{2} \stackrel{!}{=} \sigma^{2} .
$$

As one can see from (10), the principal's costs are influenced by $g$ through the terms $s \mu_{g}-\frac{\eta s^{2} \sigma_{g}^{2}}{2}$. By the definition of $\check{g}$, we have that

$$
s \mu_{\check{g}}-\frac{\eta s^{2} \sigma_{\check{g}}^{2}}{2} \leq s \mu_{g}-\frac{\eta s^{2} \sigma_{g}^{2}}{2} \forall g \in \mathcal{G} .
$$

Hence,

$$
s \mu_{\check{g}}-\frac{\eta s^{2} \sigma_{\check{g}}^{2}}{2} \leq s \sum_{\mathcal{G}} p_{g} \mu_{g}-\frac{\eta s^{2} \sum_{\mathcal{G}} p_{g} \sigma_{g}^{2}}{2} .
$$

Because with ambiguity there are at least two plausible distributions,

$$
s \sum_{\mathcal{G}} p_{g} \mu_{g}-\frac{\eta s^{2} \sum_{\mathcal{G}} p_{g} \sigma_{g}^{2}}{2}<s \sum_{\mathcal{G}} p_{g} \mu_{g}-\frac{\eta s^{2} \sum_{\mathcal{G}} p_{g}^{2} \sigma_{g}^{2}}{2} .
$$

By (20), we have that

$$
s \sum_{\mathcal{G}} p_{g} \mu_{g}-\frac{\eta s^{2} \sum_{\mathcal{G}} p_{g}^{2} \sigma_{g}^{2}}{2}=s \mu-\frac{\eta s^{2} \sigma^{2}}{2} .
$$

Hence, we have constructed a chain which shows that

$$
s \mu_{\check{g}}-\frac{\eta s^{2} \sigma_{\check{g}}^{2}}{2}<s \mu-\frac{\eta s^{2} \sigma^{2}}{2} .
$$

By (10), the principal's costs of implementing any effort $a>0$ are therefore higher with ambiguity than without.

We finally prove that the principal is strictly worse off due to ambiguity. Suppose that with ambiguity the principal optimally implements a certain effort $a^{*}>0$. Then by (2), $s^{*}=a^{*} c$. Without ambiguity, the principal can implement the same effort with lower costs; see arguments 
above. Therefore, the principal must be strictly better off without ambiguity. Suppose finally that with ambiguity the principal implements the effort $a^{*}=0$. Without ambiguity, the principal could also implement this effort and would, because of $s^{*}=0$, be equally well off than with ambiguity. But without ambiguity, she optimally implements a positive effort. The reason is that she is better off than with an effort of zero. Hence, the principal must always be better off without ambiguity. By Lemma 2 we do not have to consider the case with $s^{*}<0$.

\subsection{PROOF OF LEMMA 3}

Stochastic independence of the plausible distributions implies that

$$
\sum_{\mathcal{S}} p_{m, n} \varepsilon_{m, n} \sim N\left(\sum_{\mathcal{S}} p_{m, n} \mu_{m}, \sum_{\mathcal{S}} p_{m, n}^{2} \sigma_{n}^{2}\right)
$$

where $p_{m, n}$ is the probability assigned by the principal that the shock is drawn from the plausible distribution $[m, n], \sum_{\mathcal{S}} p_{m, n}=1$, and $p_{m, n}>0$ for all $m, n$. We assumed that aggregating the plausible distributions yields the principal's aggregated distribution which is $\varepsilon \sim N\left(0, \sigma^{2}\right)$. So

$$
\sum_{\mathcal{S}} p_{m, n} \mu_{m} \stackrel{!}{=} 0 \text { and } \sum_{\mathcal{S}} p_{m, n}^{2} \sigma_{n}^{2} \stackrel{!}{=} \sigma^{2} .
$$

The following chain shows that $\sigma^{2}<\sigma_{N}^{2}$ :

$$
\sigma^{2}=\sum_{\mathcal{S}} p_{m, n}^{2} \sigma_{n}^{2}<\sum_{\mathcal{S}} p_{m, n} \sigma_{n}^{2} \leq \sum_{\mathcal{S}} p_{m, n} \sigma_{N}^{2}=\sigma_{N}^{2}
$$

For $M \geq 2$ we can show that $\mu_{1}<0$ :

$$
0=\sum_{\mathcal{S}} p_{m, n} \mu_{m}>\sum_{\mathcal{S}} p_{m, n} \mu_{1}=\mu_{1}
$$

For $M=1$, the chain holds with equality. Therefore, $\mu_{1}=0$.

\subsection{PROOF OF PROPOSITIONS 4 AND 5}

Step 1. The agent's belief about the distribution of $\varepsilon_{Z}$ is characterized by $\left(\mu_{Z, m}, \sigma_{Z, n}^{2}\right)$. Simple calculations yield that the agent's certainty equivalent is

$\hat{w}(\cdot)=t+\left(s_{Y}+s_{Z}\right) a+s_{Z} \mu_{Z, m}-\frac{1}{2} \eta\left(s_{Y}^{2} \sigma_{Y}^{2}+2 \rho s_{y} s_{Z} \sigma_{y} \sigma_{Z, n}+s_{Z}^{2} \sigma_{Z, n}^{2}\right)-\frac{1}{2} c a^{2}$. 
The principal's maximization problem can be simplified to

$$
\begin{array}{r}
\max _{s_{Y}, s_{Z}} \frac{s_{Y}+s_{Z}}{c}+s_{Z} \mu_{Z, m}-\frac{1}{2} \eta\left(s_{Y}^{2} \sigma_{Y}^{2}+2 \rho s_{y} s_{Z} \sigma_{y} \sigma_{Z, n}+s_{Z}^{2} \sigma_{Z, n}^{2}\right) \\
-\frac{1}{2} \frac{\left(s_{Y}+s_{Z}\right)^{2}}{c}-\bar{w} .
\end{array}
$$

The derivatives are

$$
\begin{aligned}
& \frac{d \ldots}{d s_{Y}}=\frac{1}{c}-\eta\left(s_{Y} \sigma_{Y}^{2}+s_{z} \rho \sigma_{Y} \sigma_{Z, n}\right)-\frac{s_{Y}+s_{Z}}{c} \\
& \frac{d \ldots}{d s_{Z}}=\frac{1}{c}-\eta\left(s_{Z} \sigma_{Z, n}^{2}+s_{Y} \rho \sigma_{Y} \sigma_{Z, n}\right)-\frac{s_{Y}+s_{Z}}{c}+\mu_{Z, m} .
\end{aligned}
$$

Because an optimum exists and $s_{y}$ can be set freely, in the optimum $d \ldots / d s_{Y}=0$. Hence,

$$
s_{Y}=\frac{1-s_{Z} \rho \eta c \sigma_{Y} \sigma_{Z, n}-s_{Z}}{1+\eta c \sigma_{Y}^{2}} .
$$

Plugging (25) into (23) yields

$$
\begin{array}{r}
\frac{d \ldots}{d s_{Z}}=\frac{1}{c}-\eta\left(s_{Z} \sigma_{Z, n}^{2}+\frac{1-s_{Z} \rho \eta c \sigma_{Y} \sigma_{Z, n}-s_{Z}}{1+\eta c \sigma_{Y}^{2}} \rho \sigma_{Y} \sigma_{Z, n}\right) \\
-\frac{\frac{1-s_{Z} \rho \eta c \sigma_{Y} \sigma_{Z, n}-s_{Z}}{1+\eta c \sigma_{Y}^{2}}+s_{Z}}{c}+\mu_{Z, m}
\end{array}
$$

which can be rewritten as

$$
\begin{aligned}
\frac{d \ldots}{d s_{Z}} c=1 & -\frac{1+\rho \eta c \sigma_{Y} \sigma_{Z, n}}{1+\eta c \sigma_{Y}^{2}}+\mu_{Z, m} c \\
& +s_{Z}\left[-\eta c \sigma_{Z, n}^{2}-1+\left(1+\rho \eta c \sigma_{Y} \sigma_{Z, n}\right) \frac{1+\rho \eta c \sigma_{Y} \sigma_{Z, n}}{1+\eta c \sigma_{Y}^{2}}\right]
\end{aligned}
$$

Because

$$
[\cdot]\left(1+\eta c \sigma_{Y}^{2}\right)=-\left(1+\eta c \sigma_{Y}^{2}\right)\left(1+\eta c \sigma_{Z, n}^{2}\right)+\left(1+\rho \eta c \sigma_{Y} \sigma_{Z, n}\right)^{2} \leq 0
$$

the square bracket $[\cdot]$ of $(26)$ is non-positive.

Step 2. From (22) one can see that for $\rho \leq 0$ setting $s_{z}<0$ cannot be optimal. Prove by contradiction. Assume the contrary $\tilde{s_{Z}}<0$ and $\tilde{s_{Y}}$ are optimal. We must have $\tilde{s_{Z}}+\tilde{s}_{Y}>0$, otherwise $s_{Y}=s_{Z}=0$ dominates. By setting $s_{Z}=0$ and $s_{y}=\tilde{s_{Z}}+\tilde{s_{Y}}>0$ dominates $\left(\tilde{s_{Z}}<0, \tilde{s_{Y}}\right)$. A contradiction.

Step 3. What plausible distribution does the agent use to evaluate a wage scheme? Observe that for $s_{z} \rightarrow 0 s_{Y}>0$. From (21) we see that 
for $s_{Z} \searrow 0$ we have: $\mu_{Z, m}=\mu_{Z, 1}$; for $\rho>0, \sigma_{Z, n}^{2}=\sigma_{Z, N}^{2}$; for $\rho=0$, $\sigma_{Z, n}^{2}=\sigma_{Z, N}^{2}$; for $\rho<0, \sigma_{Z, n}^{2}=\sigma_{Z, 1}^{2}$. From (21) we see that for $s_{Z} \nearrow 0$ we have: $\mu_{Z, m}=\mu_{Z, M}$; for $\rho>0, \sigma_{Z, n}^{2}=\sigma_{Z, 1}^{2}$; for $\rho=0, \sigma_{Z, n}^{2}=\sigma_{Z, N}^{2}$; for $\rho<0, \sigma_{Z, n}^{2}=\sigma_{Z, N}^{2}$.

Step 4. Case 1: $\rho<0$. From Step 2 we know that $s_{Z} \geq 0$. Because the square bracket of (26) is non-positive (see Step 1), for fixed $\left(\mu_{Z, m}, \sigma_{Z, n}^{2}\right)$, the principal wants to set $s_{Z}>0$ if and only if $\left.\frac{d \ldots}{d s_{Z}}\right|_{s_{z} \searrow 0}>0$. Hence, with (26) and Step 3 this implies that $s_{Z}>0$ if and only if

$$
\mu_{Z, 1}>\frac{\eta \sigma_{Y}\left(\rho \sigma_{Z, 1}-\sigma_{Y}\right)}{1+\eta c \sigma_{Y}^{2}}
$$

otherwise $s_{Z}=0$.

Case 2: $\rho=0$. Similar steps as before yield that $s_{Z}>0$ if and only if

$$
\mu_{Z, 1}>\frac{-\eta \sigma_{Y}^{2}}{1+\eta c \sigma_{Y}^{2}}
$$

otherwise $s_{Z}=0$.

Case 3: $\rho>0$. Because the square bracket of (26) is non-positive (see Step 1), for fixed $\left(\mu_{Z, m}, \sigma_{Z, n}^{2}\right)$, the principal wants to set $s_{Z}>0$ instead of $s_{Z}=0$ if and only if $\left.\frac{d \ldots}{d s_{Z}}\right|_{s_{z} \searrow 0}>0$. Hence, with (26) and Step 3 this implies that $s_{Z}>0$ is preferred over $s_{Z}=0$ if and only if

$$
\mu_{Z, 1}>\frac{\eta \sigma_{Y}\left(\rho \sigma_{Z, N}-\sigma_{Y}\right)}{1+\eta c \sigma_{Y}^{2}}
$$

Similarly, $s_{Z}<0$ is preferred over $s_{Z}=0$ if and only if

$$
\mu_{Z, M}<\frac{\eta \sigma_{Y}\left(\rho \sigma_{Z, 1}-\sigma_{Y}\right)}{1+\eta c \sigma_{Y}^{2}} .
$$

Step 5. Up to now we have only used local arguments for $s_{Z} \rightarrow 0$. Are these arguments globally valid? First, if the local analysis predicts that the principal wants to set $s_{z} \neq 0$, then this is obviously also globally true. Second, if the local analysis predicts the contrary, namely that in the optimum $s_{Z}=0$, it becomes more difficult. For fixed $\left(\mu_{Z, m}, \sigma_{Z, n}^{2}\right)$ we see from (26) that when $\left.\frac{d \ldots}{d s_{Z}}\right|_{s_{z} \searrow 0} \leq 0$ than also $\left.\frac{d \ldots}{d s_{Z}}\right|_{s_{z}>0} \leq 0$ and when $\left.\frac{d \ldots}{d s_{Z}}\right|_{s_{z} \nearrow_{0}} \geq 0$ than also $\left.\frac{d \ldots}{d s_{Z}}\right|_{s_{z}<0} \geq 0$. This implies that our local results are globally valid for fixed $\left(\mu_{Z, m}, \sigma_{Z, n}^{2}\right)$. However, $\left(\mu_{Z, m}, \sigma_{Z, n}^{2}\right)$ may change with different $\left(s_{Y}, s_{Z}\right)$. But this does not change our results: the value of the principal's objective function for a fixed $\left(\mu_{Z, m}, \sigma_{Z, n}^{2}\right)$ is an upper bound for the objective function when $\left(\mu_{Z, m}, \sigma_{Z, n}^{2}\right)$ are chosen so that (21) 
is minimized (which is true because of ambiguity aversion). Hence, when the principal prefers $s_{Z}=0$ for a fixed $\left(\mu_{Z, m}, \sigma_{Z, n}^{2}\right)$, she prefers $s_{Z}=0$ also when $\left(\mu_{Z, m}, \sigma_{Z, n}^{2}\right)$ are chosen so that $(21)$ is minimized.

\section{LITERATURE}

Baker, George P., Michael C. Jensen, and Kevin J. Murphy (1988). Compensation and Incentives: Practise vs. Theory. Journal of Finance 43, 593-616.

Billot, Antoine, Alain Chateauneuf, Itzhak Gilboa, and Jean-Marc Tallon (2000). Sharing Beliefs: Between Agreeing and Disagreeing. Econometrica 68, 685-694.

Bolton, Patrick and Mathias Dewatripont (2005). Contract Theory. Boston: MIT Press.

Cramér, Harald (1936). Über eine Eigenschaft der normalen Verteilungsfunktion. Mathematische Zeitschrift 41, 405-414.

De la Rosa, Leonidas E. (2007). Overconfidence and Moral Hazard. Danish Centre for Accounting and Finance, Working Paper No. 24.

DellaVigna, Stefano and Ulrike Malmendier (2004). Contract Design and Self-Control: Theory and Evidence. Quarterly Journal of Economics 119, 353-402.

Dow, James and Sérgio Ribeiro Da Costa Werlang (1992). Uncertainty Aversion, Risk Aversion, and the Optimal Choice of Portfolio. Econometrica 60, 197-204.

Ellsberg, Daniel (1961). Risk, ambiguity, and the Savage axioms. Quarterly Journal of Economics 75, 643-669.

Epstein, Larry G. and Tan Wang (1994). Intertemporal Asset Pricing Under Knightian Uncertainty. Econometrica 62, 283-322.

Fang, Hanming and Giuseppe Moscarini (2005). Morale Hazard. Journal of Monetary Economics 52, 749-777.

Feltham, Gerald A. and Jim Xie (1994). Performance Measure Congruity and Diversity in Multi-Task Principal/Agent Relations. Accounting Review 69, 429-453.

Ghirardato, Paolo (1994). Agency Theory with Non-Additive Uncertainty. Mimeo.

Ghirardato, Paolo, Fabio Maccheroni, and Massimo Marinacci (2004). Differentiating Ambiguity and Ambiguity Attitude. Journal of Economic Theory 118, 133-173.

Gilboa, Itzhak, Andrew W. Postlewaite, and David Schmeidler (2008). Probability and Uncertainty in Economic Modeling. Journal of Economic Perspectives 22, 173188.

Gilboa, Itzhak and David Schmeidler (1989). Maxmin Expected Utility with NonUnique Prior. Journal of Mathematical Economics 18, 141-153.

Holmström, Bengt (1979). Moral Hazard and Observability. Bell Journal of Economics 10, 74-91.

Holmström, Bengt and Paul Milgrom (1987). Aggregation and Linearity in the Provision of Intertemporal Incentives. Econometrica 55, 303-328.

Holmström, Bengt and Paul Milgrom (1991). Multitask Principal Agent Analyses: Incentive Contracts, Asset Ownership and Job Design. Journal of Law, Economics, and Organization 7 (special issue), 24-52.

Hvide, Hans K. (2002). Pragmatic Beliefs and Overconfidence. Journal of Economic Behavior $\&$ Organization 48, 15-28. 
Jensen, Michael C. and Kevin J. Murphy (1990). Performance Pay and Top-Management Incentives. Journal of Political Economy 98, 225-264.

Lang, Matthias (2007). Ambiguity Aversion in a Multitask Moral Hazard Framework. Mimeo.

Karni, Edi (2009). A Reformulation of the Maxmin Expected Utility Model with Application to Agency Theory. Journal of Mathematical Economics 45, 97-112

Kellner, Christian (2009). Tournaments as Response to Ambiguity Aversion in Incentive Contracts. Mimeo.

Klibanoff, Peter, Massimo Marinacci, and Sujoy Mukerji (2005). A Smooth Model of Decision Making Under Ambiguity. Econometrica 73, 1849-1892.

Maccheroni, Fabio, Massimo Marinacci, and Aldo Rustichini (2006). Ambiguity Aversion, Robustness, and the Variational Representation of Preferences. Econometrica $74,1447-1498$.

Mukerji, Sujoy (1998). Ambiguity Aversion and Incompleteness of Contractual Form. American Economic Review 88, 1207-1231.

Mukerji, Sujoy (2003). Ambiguity Aversion and Cost-Plus Procurement Contracts. University of Oxford, Department of Economics Discussion Paper 171.

Offerman, Theo, Joep Sonnemans, Gijs van de Kuilen, and Peter P. Wakker (2009). A Truth Serum for Non-Bayesians: Correcting Proper Scoring Rules for Risk Attitudes. Review of Economic Studies 76, 1461-1489.

Post, Thierry, Martijn J. van den Assem, Guido Baltussen, and Richard H. Thaler (2008). Deal or No Deal? Decision Making under Risk in a Large-Payoff Game Show. American Economic Review 98, 38-71.

Prendergast, Canice (1999). The Provision of Incentives in Firms. Journal of Economic Literature 37, 7-63.

Rustichini, Aldo (2005). Emotion and Reason in Making Decisions. Science 310, 1624-1625.

Santos-Pinto, Luís (2008). Positive Self-Image and Incentives in Organizations. Economic Journal 118, 1315-1332.

Savage, Leonard (1954). The Foundations of Statistics. New York: John Wiley. 